Check for updates

Cite this: Nanoscale Adv., 2019, 1, 2157

Received 13th March 2019

Accepted 14th April 2019

DOI: 10.1039/c9na00157c

rsc.li/nanoscale-advances

\section{From a precursor to an etchant: spontaneous inversion of the role of Au(III) chloride for one-pot synthesis of smooth and spherical gold nanoparticles $\uparrow$}

\author{
Seokyoung Yoon, (D) a Chansong Kim, ${ }^{\mathrm{b}}$ Byoungsang Lee (DD ${ }^{\mathrm{b}}$ \\ and Jung Heon Lee (D) *abc
}

We report the inversion of the role of $A u(I I)$ chloride, from a gold precursor to an etchant, for the synthesis of smooth and spherical AuNPs with nanoscale size tunability in a one-pot-system. Inversion of the role of $\mathrm{Au}(\mathrm{III})$ chloride was achieved by regulating the ratio between the reducing agent and $\mathrm{Au}(\mathrm{III})$ chloride.

\section{Introduction}

Among metal nanoparticles, gold nanoparticles (AuNPs) have been actively studied because of their high biocompatibility, stability, and excellent catalytic, electrical, and optical properties. $^{1-6}$ In particular, the localized surface plasmon resonance (LSPR) of AuNPs allows them to be used for diverse optical applications, including sensing, ${ }^{7-9}$ surface-enhanced Raman scattering, ${ }^{10}$ surface-enhanced fluorescence, ${ }^{\mathbf{1 1}}$ and plasmon resonance energy transfer. ${ }^{12}$ It is widely known that the LSPR of AuNPs is greatly affected by their size, shape, and morphology. Thus, numerous studies have been conducted to control these factors. ${ }^{13-15}$ However, AuNPs synthesized via bottom-up strategies based on wet chemistry usually have faceted structures and are poly-crystalline and polydisperse in size and shape. ${ }^{\mathbf{1 6}}$

Recently, single-crystalline, smooth and round AuNPs have been developed with uniform optical properties. For instance, Xia et al. reported the synthesis of single-crystalline spherical AuNPs via a seed-mediated method. ${ }^{17,18} \mathrm{Yi}$ et al. reported the preparation of highly spherical and monocrystalline AuNPs using a cyclic process. ${ }^{19}$ In addition, Mirkin and co-workers

${ }^{a}$ SKKU Advanced Institute of Nanotechnology (SAINT), Sungkyunkwan University (SKKU), Suwon 16419, Republic of Korea. E-mail: jhlee7@skku.edu; Fax: +82-502302-1918; Tel: +82-31-290-7404

${ }^{b}$ School of Advanced Materials Science and Engineering, Sungkyunkwan University (SKKU), Suwon 16419, Republic of Korea

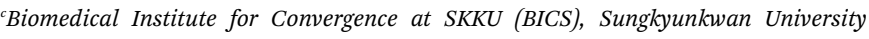
(SKKU), Suwon 16419, Republic of Korea

$\dagger$ Electronic supplementary information (ESI) available. See DOI: $10.1039 / \mathrm{c} 9 \mathrm{na} 00157 \mathrm{c}$ synthesized universal AuNP seeds through iterative reduction and oxidation processes. ${ }^{20}$ Notably, even nanoscale changes in the gap size can considerably change the interparticle coupling, leading to dramatic variation in the electromagnetic coupling of clustered metamolecules and superlattice metamaterials. ${ }^{21,22}$ Thus, fine size-tunability of AuNPs over the nanometer scale is very important.

We herein present a novel strategy to synthesize smooth and highly spherical AuNPs with nanometer scale tunability and monodispersity through a facile and reproducible etching method. It should be pointed out that this process is induced by spontaneous inversion of the role of $\mathrm{Au}(\mathrm{III})$ chloride $\left(\mathrm{AuCl}_{4}{ }^{-}\right.$) from a precursor into an etchant without the introduction of an additional etchant.

In general, researchers have used various types of etchants to control the shape and size of AuNPs. ${ }^{23-25}$ In this study, we used a $\mathrm{AuCl}_{4}{ }^{-}$-cetyltrimethylammonium chloride (CTAC) complex for the oxidative etching of AuNPs, which was previously reported by Halas et al. ${ }^{26}$ and studied in detail by Liz-Marzán et $a l .{ }^{27}$ In our study, gold sources can act as a gold precursor during the initial AuNP synthesis process and as an etchant during the subsequent etching process. The exchange of the role of $\mathrm{AuCl}_{4}{ }^{-}$was achieved by controlling the molar ratio between the reducing agent and $\mathrm{AuCl}_{4}{ }^{-}$. In addition, we found that a balance between the surface diffusion rate and etching rate of $\mathrm{Au}$ atoms on the AuNP surface is very important for the subsequent etching process to induce a size decrease without any morphological transformation. We could fine-tune the size of AuNPs in a facile manner by extracting the samples in a timely sequence, similar to that reported for quantum dots. ${ }^{28}$

\section{Results and discussion}

We synthesized AuNPs through a seed-mediated and stepwise growth process (Fig. 1). ${ }^{18}$ By rapidly reducing the gold precursor $\left(\mathrm{AuCl}_{4}{ }^{-}\right)$with sodium borohydride $\left(\mathrm{NaBH}_{4}\right)$ in the presence of cetyltrimethylammonium bromide (CTAB), we synthesized single-crystalline gold clusters (Au-clusters). Subsequently, an 


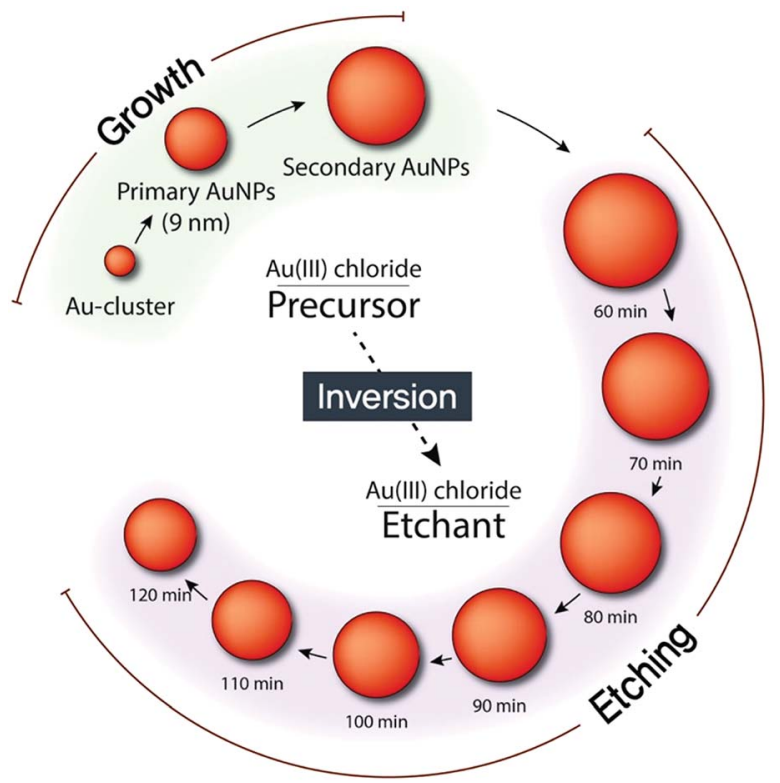

Fig. 1 Schematic of stepwise growth and etching of AuNPs with Au(III) chloride functioning as a precursor and etchant.

ascorbic acid (AA) solution was injected, as a reductant, into the mixture containing CTAC, Au-clusters, and $\mathrm{AuCl}_{4}{ }^{-}$to synthesize primary AuNPs. Thus, Au-clusters functioned as seeds for the synthesis of primary AuNPs with CTAC ligands. For larger AuNPs, denoted as secondary AuNPs, primary AuNPs were used as seeds instead of Au-clusters. Accordingly, the gold source solution was slowly injected into the mixture of primary AuNPs, CTAC, and AA to synthesize secondary AuNPs. Final sizes of both primary and secondary AuNPs were found to be inversely proportional to the amount of seeds used. To obtain smooth and spherical AuNPs, the growth must be controlled by thermodynamics rather than kinetics. ${ }^{\mathbf{2 9}, 30}$ Hence, we used CTAC instead of $\mathrm{CTAB}$ in which bromide ions have strong binding affinity to $\mathrm{Au}$ atoms and ions as a capping agent, because its weak binding affinity to the AuNP surface and the gold ion enables the balance between surface diffusion of gold atoms and gold deposition on the AuNP surface. ${ }^{31}$ Furthermore, the capping of AuNPs with CTAC is highly beneficial: CTAC can be easily replaced with other ligands for further applications. ${ }^{31}$ CTAC capped AuNPs can also be an ideal building block for selfassembly and potential drug carriers. ${ }^{31,32}$

For the feasibility of the synthesis, we conducted the whole process at room temperature $\left(25^{\circ} \mathrm{C}\right)$. The surface diffusion rate is mainly dependent on the temperature, and for gold atoms, it is sufficiently high at room temperature. ${ }^{17,33-35}$ Thus, we tried to control the rate of gold deposition by adjusting the amount of AA to attain an optimum value, at which smooth spherical AuNPs would be formed. Fig. 2a shows the transmission electron microscopy (TEM) image of the primary AuNPs. As observed, the primary AuNPs are highly monodisperse with a size of $9.2 \mathrm{~nm}$. UV-vis spectroscopy data are presented in Fig. S2a $\uparrow$ magenta line. Notably, when primary AuNPs were further reduced with different amounts of AA, secondary AuNPs with different morphologies were produced (see Fig. 2b-d).

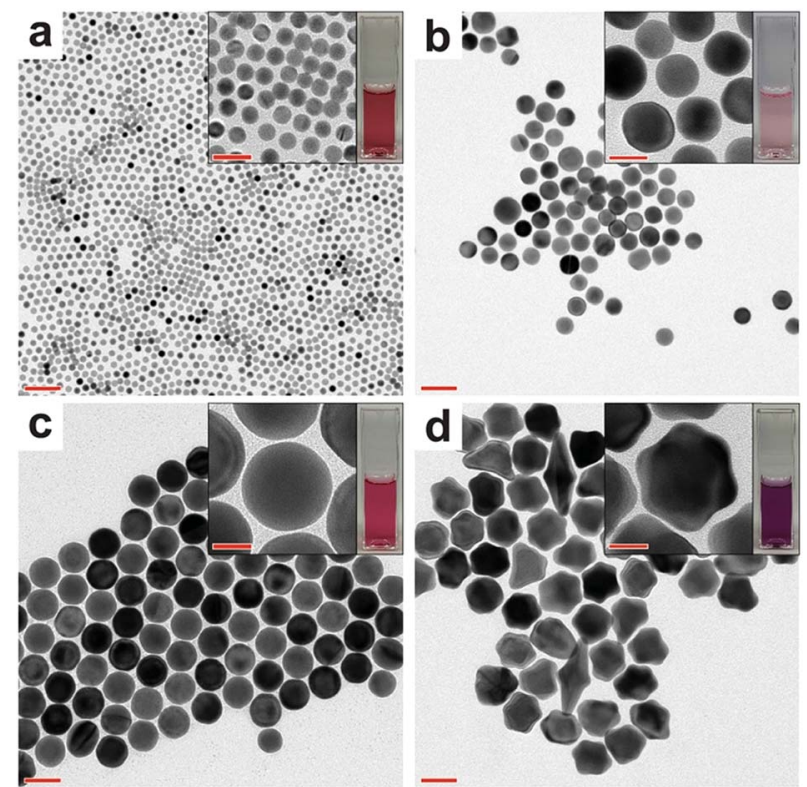

Fig. 2 TEM images of (a) primary AuNPs and secondary AuNPs prepared with (b) 60 , (c) 110, and (d) $160 \mu \mathrm{L}$ of AA. Scale bar: $50 \mathrm{~nm}$; inset scale bar: $20 \mathrm{~nm}$.

When $60 \mu \mathrm{L}$ of AA was used, the size distribution was broad because of the insufficient amount of the reducing potential, as shown in Fig. $2 \mathrm{~b}$ and S1a $\dagger$ (scanning electron microscopy (SEM) image). On the other hand, when $160 \mu \mathrm{L}$ of AA was used, branched AuNPs were formed (Fig. 2d and S1c $\dagger$ ). Because the presence of excessive AA led to over-deposition of gold atoms on the AuNPs - with specific crystal-growth directions, it was hard to achieve a balance between gold deposition and gold surface diffusion on the AuNP surface. Consequently, AuNPs did not grow spherically under this condition. In contrast, 110 $\mu \mathrm{L}$ of AA was found to be appropriate to achieve a balanced rate between gold deposition and surface diffusion, and in turn, spherical secondary AuNPs were obtained (Fig. 2c and S1b $\dagger$ ). Thus, if a reductant that has either stronger or weaker reducing power than AA was used, the shape of the AuNPs will become different and the amount of reducing agent needed to synthesize spherical AuNPs will be significantly varied. To confirm the uniformity of the synthesized AuNPs, we analysed them with UV-vis spectroscopy and Dynamic Light Scattering (DLS) (Fig. S $2 \dagger$ ). Only a single DLS peak was observed for the spherical secondary AuNPs. On the other hand, the DLS spectra of the branched AuNPs showed double peaks attributed to the rotational diffusion mode arising from their anisotropic shape (Fig. S2b †). ${ }^{36,37}$

When $110 \mu \mathrm{L}$ of AA was used, we found that the size of the secondary AuNPs slowly decreased, while the spherical shape was retained as the reaction progressed. Thus, by extracting samples in a timely sequence from the same batch, we were able to obtain smooth AuNPs with $1 \mathrm{~nm}$-scale size tunability (Fig. 3). We identified that the crystalline structure of AuNPs, which went through the etching process, retained the inherent spherical single crystal structure, as approved by highresolution TEM (Fig. 3b). This etching process is proved by 
a

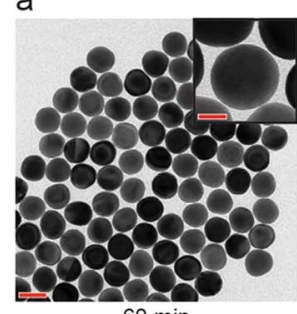

$60 \mathrm{~min}$

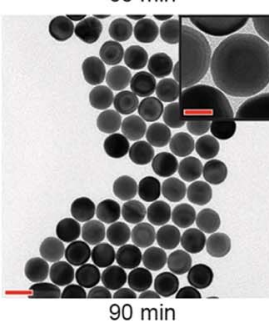

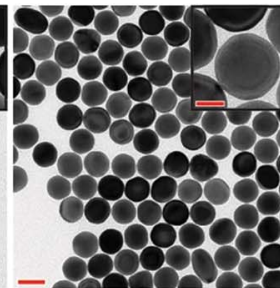
$70 \mathrm{~min}$

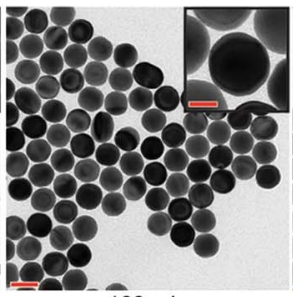

$100 \mathrm{~min}$

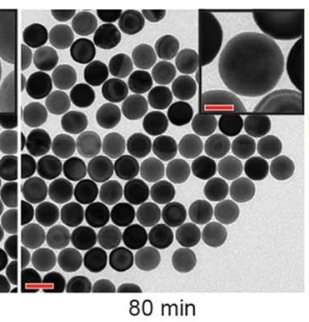

b
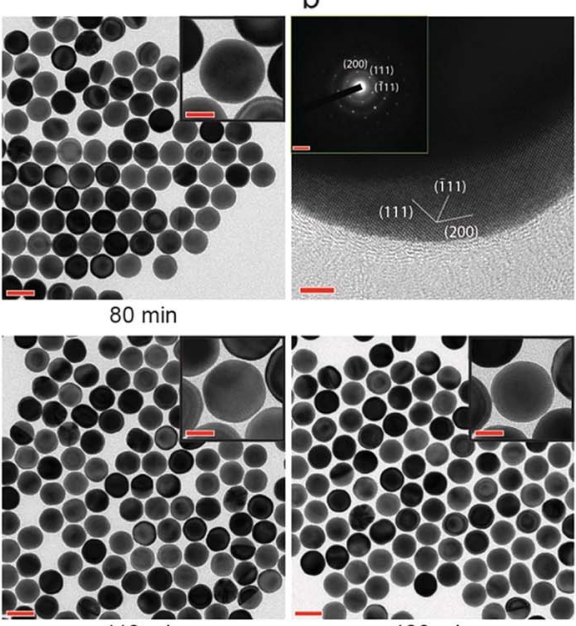

$110 \mathrm{~min}$

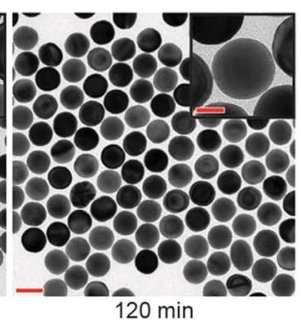

C

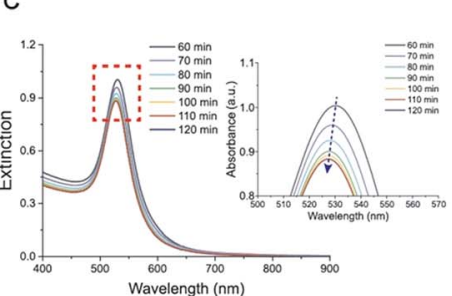

d

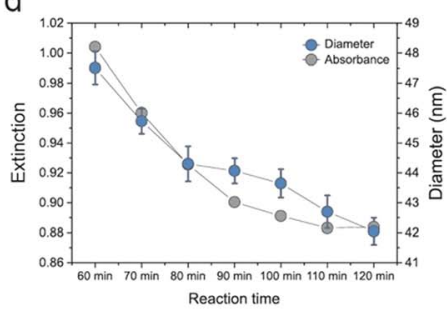

Fig. 3 Variation in size and UV-vis spectra of secondary AuNPs as a function of reaction time. (a) TEM images of secondary AuNPs with reaction time varying from $60 \mathrm{~min}$ to $120 \mathrm{~min}$. Scale bar: $50 \mathrm{~nm}$; inset scale-bar: $20 \mathrm{~nm}$. (b) Selected area electron diffraction pattern for individual secondary AuNPs (reaction time: 60 min). (c) UV-vis spectra of secondary AuNPs with different reaction times. (d) Variation in the extinction and diameter of secondary AuNPs as a function of reaction time.

the decrease of extinction at $400 \mathrm{~nm}$ which presents the concentration of $\mathrm{Au}(0)$ (Fig. 3c). ${ }^{27}$ The intensity of the absorbance peak is affected by the concentration and size of the AuNPs. ${ }^{17}$ In this experiment, the number of AuNPs is kept identical due to the restriction of nucleation at the growth stage. Thus, the variation in the intensity of extinction and size of the AuNPs is related. The intensity of the UV-vis spectra decreased as the etching proceeded, indicating a decrease in the size of the AuNPs (Fig. 3c and d). In addition, the AuNPs showed absorbance values comparable to those calculated on the basis of Mie theory (Fig. S3 $\uparrow$ ). This confirmed the formation of smooth and spherical AuNPs with high monodispersity.

We hypothesized that unreduced $\mathrm{AuCl}_{4}{ }^{-}$ions induced etching of the AuNPs, because of the lack of AA and the variation of the molar ratio between $\mathrm{AA}$ and $\mathrm{AuCl}_{4}{ }^{-}\left([\mathrm{AA}] /\left[\mathrm{AuCl}_{4}{ }^{-}\right]\right)$occurring across the reaction (Fig. S4, $\dagger$ schematic of the AuNP-etching model). According to the stoichiometric reduction reaction of gold, an $[\mathrm{AA}] /\left[\mathrm{AuCl}_{4}{ }^{-}\right]$ratio of 1.5 was required to fully reduce $\mathrm{AuCl}_{4}{ }^{-}$to $\mathrm{Au}(0)$. For $110 \mu \mathrm{L}$ of AA, $[\mathrm{AA}] /\left[\mathrm{AuCl}_{4}{ }^{-}\right]$corresponded to 1.1. Thus, it was not sufficient to fully reduce the whole $\mathrm{AuCl}_{4}{ }^{-}$in the growth solution, resulting in the eventual etching of the AuNPs. In addition, $[\mathrm{AA}] /\left[\mathrm{AuCl}_{4}{ }^{-}\right]$continuously changed because the $\mathrm{AuCl}_{4}{ }^{-}$solution was slowly injected into the growth solution, instead of a one-shot injection. In this sense, $[\mathrm{AA}] /\left[\mathrm{AuCl}_{4}{ }^{-}\right]$in the latter part of the reaction is relatively smaller than the ratio in the former part of the reaction, which leads to the retardation of the reduction reaction.

The reduction of $\mathrm{AuCl}_{4}{ }^{-}$in the presence of AA occurs in two steps. In the intermediate stage of the reduction of $\mathrm{AuCl}_{4}{ }^{-}$to $\mathrm{Au}(0), \mathrm{AuCl}_{2}{ }^{-}$is formed because of the low reducing power of AA. ${ }^{16}$ To prove our hypothesis, we monitored the concentrations of $\mathrm{AuCl}_{4}{ }^{-}$and $\mathrm{AuCl}_{2}{ }^{-}$over time using UV-vis spectroscopy (Fig. 4). We separately measured the extinctions of $\mathrm{AuCl}_{4}{ }^{-}$ CTAC and $\mathrm{AuCl}_{2}{ }^{-}$-CTAC complexes, to monitor the amounts of $\mathrm{AuCl}_{4}{ }^{-}$and $\mathrm{AuCl}_{2}{ }^{-}$, respectively. Notably, the inherent absorbances of $\mathrm{AuCl}_{4}{ }^{-}$and $\mathrm{AuCl}_{2}{ }^{-}$without CTAC were different from those with CTAC (see the UV-vis spectra of CTAC and $\mathrm{AuCl}_{4}{ }^{-}$in Fig. S5a†). For the yellowish $\mathrm{AuCl}_{4}{ }^{-}$-CTAC complex, two peaks were observed at $228 \mathrm{~nm}$ and $321 \mathrm{~nm}$. The extinction at $321 \mathrm{~nm}$ contributed to the yellowish color, as both the color and extinction at $321 \mathrm{~nm}$ disappeared after the addition of AA (Fig. 4a). On the other hand, for the $\mathrm{AuCl}_{2}{ }^{-}$-CTAC complex, which was transparent under ambient light, a distinct peak at $243 \mathrm{~nm}$ was observed. These results indicated that the amounts of $\mathrm{AuCl}_{4}{ }^{-}$-CTAC and $\mathrm{AuCl}_{2}{ }^{-}$-CTAC complexes in the AuNP solution mixture could be separately measured. To monitor the exact concentrations of $\mathrm{AuCl}_{4}{ }^{-}$-CTAC and $\mathrm{AuCl}_{2}{ }^{-}$-CTAC complexes, we separated the as-synthesized solution (AS) into supernatant (SP) and nanoparticle solutions (NP) and measured the extinctions of $\mathrm{AuCl}_{4}{ }^{-}$-CTAC and $\mathrm{AuCl}_{2}{ }^{-}$-CTAC complexes individually (Fig. S5b $\dagger$ ).

In the initial stage of the growth, two extinction peaks were observed at $541 \mathrm{~nm}$ and $695 \mathrm{~nm}$ for AuNPs reduced for $20 \mathrm{~min}$
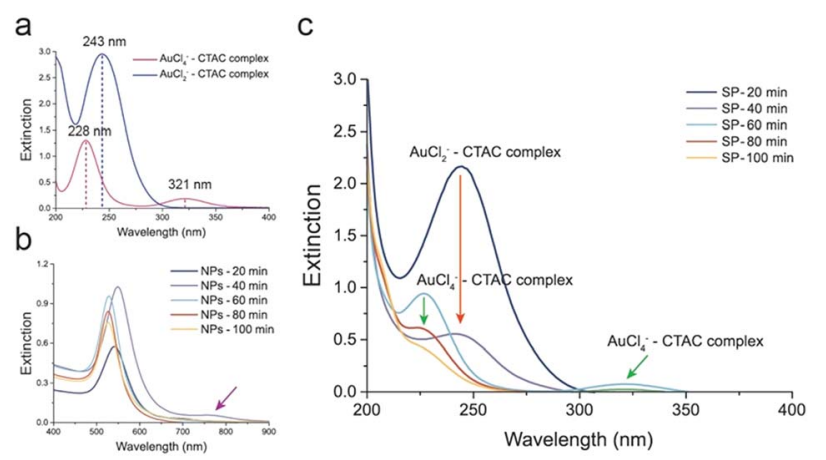

Fig. 4 Mechanism of $\mathrm{AuCl}_{4}{ }^{-}-\mathrm{CTAC}$-derived AuNP etching. (a) UV-vis spectra of $\mathrm{AuCl}_{4}{ }^{-}-\mathrm{CTAC}$ and $\mathrm{AuCl}_{2}{ }^{-}-\mathrm{CTAC}$ complexes. (b) UV-vis spectra of NP obtained from AS with different reaction times. (c) UV-vis spectra of SP obtained from AS with different reaction times. 
(NPs-20 min) and at $549 \mathrm{~nm}$ and $760 \mathrm{~nm}$ for AuNPs reduced for $40 \mathrm{~min}$ (NPs-40 min) (see Fig. 4b). This indicates the formation of anisotropic AuNPs with a branched morphology, similar to the case of gold nanorods previously reported. ${ }^{38}$ However, once the growth proceeded due to the surface diffusion of gold atoms, smooth and spherical AuNPs were formed, and a single extinction peak was observed at 528, 526, and $526 \mathrm{~nm}$ for AuNPs reduced for 60, 80, and $100 \mathrm{~min}$, respectively (Fig. 4b and S6†). After $20 \mathrm{~min}$ of the growth, the characteristic extinction peak of the $\mathrm{AuCl}_{2}{ }^{-}$-CTAC complex appeared at $243 \mathrm{~nm}$. The peak intensity decreased within $40 \mathrm{~min}$ of growth because of the reduction of the injected $\mathrm{AuCl}_{4}{ }^{-}$-CTAC complex by AA in the solution, and its consumption, resulting in the deposition on AuNPs (Fig. 4c, red arrow). After $60 \mathrm{~min}$ of the reaction, the $\mathrm{AuCl}_{4}{ }^{-}$-CTAC complex extinction peaks emerged at $228 \mathrm{~nm}$ and $321 \mathrm{~nm}$, indicating the inversion of the role of $\mathrm{AuCl}_{4}{ }^{-}$from a gold precursor into an etchant.

As AA was consumed during the reduction reaction, AA became insufficient in the latter part of the reaction. Thus, retardation of reduction of the $\mathrm{AuCl}_{4}{ }^{-}$-CTAC complex and etching of AuNPs could have happened. Although the amount of AA left was small, the intensity of the $\mathrm{AuCl}_{4}{ }^{-}$-CTAC complex extinction peaks continuously decreased because slow reduction reaction continued to progress (60-100 min in Fig. 4c). In consequence, the injected $\mathrm{AuCl}_{4}{ }^{-}$was found to play timedependent multiple roles: a gold precursor in the beginning of the reaction and an etchant in the end.

The role of $\mathrm{AuCl}_{4}{ }^{-}$as an etchant depended on the retardation of the reduction reaction caused by the lack of AA. We varied the amount of AA to check its role in detail during the synthesis of secondary AuNPs. The extinction peak showed that the retardation of reduction depended on the amount of AA, as expected. When a small amount of AA was used $(70 \mu \mathrm{L})$, relatively high-intensity extinction peaks of the $\mathrm{AuCl}_{4}{ }^{-}$-CTAC complex emerged early in the reaction indicating early inversion of $\mathrm{AuCl}_{4}{ }^{-}$into an etchant (40 min in Fig. S7a $\dagger$ ). As the etching rate was very high under this condition, there was no balance between etching and diffusion, which resulted in the formation of non-spherical AuNPs (Fig. S7c $\dagger$ ). However, the extinction peaks of the $\mathrm{AuCl}_{4}{ }^{-}$-CTAC complex did not appear when a large amount of AA was used $(130 \mu \mathrm{L})$, indicating the absence of inversion (Fig. S7b and $c^{\dagger}$ ). Through these data, we confirmed that an optimum amount of AA is a prerequisite to change the role of the $\mathrm{AuCl}_{4}{ }^{-}$-CTAC complex: from a gold precursor to an etchant in the one-pot system. In addition, we prepared large AuNPs in the range 70-80 nm using the same method (Fig. S8 $\dagger$ ), as these AuNPs with considerably large scattering cross-section can be used for diverse optical applications.

\section{Conclusions}

In summary, we successfully demonstrated that the size of smooth and spherical AuNPs could be fine-tuned across various size ranges through etching in a one-pot system. Notably, we found that the role of $\mathrm{AuCl}_{4}{ }^{-}$could be changed: from a gold precursor to an etchant, depending on the amount of the reducing agent. A balance between the chemical etching rate and surface diffusion rate was found to be critical to maintain the spherical shape of AuNPs. As demonstrated, this strategy is a simple and powerful method to control the size of highly uniform AuNPs, and it can be used for various applications, such as plasmonic coupling and optical interference between AuNPs and fluorescent dye molecules, depending on the size of the AuNPs.

\section{Conflicts of interest}

There are no conflicts to declare.

\section{Acknowledgements}

This research was supported by the National Research Foundation of Korea (NRF) grants funded by the Ministry of Science and ICT for the Bio-inspired Innovation Technology Development Project (NRF-2018M3C1B7021997) and a grant funded by the Ministry of Education for Basic Science Research Program (NRF-2018R1D1A1B07043213).

\section{Notes and references}

1 H. Chen, L. Shao, Q. Li and J. Wang, Chem. Soc. Rev., 2013, 42, 2679-2724.

2 X. Yang, M. Yang, B. Pang, M. Vara and Y. Xia, Chem. Rev., 2015, 115, 10410-10488.

3 Y. Mikami, A. Dhakshinamoorthy, M. Alvaro and H. García, Catal. Sci. Technol., 2013, 3, 58-69.

4 E. C. Dreaden, A. M. Alkilany, X. Huang, C. J. Murphy and M. A. El-Sayed, Chem. Soc. Rev., 2012, 41, 2740-2779.

5 J. H. Joo, B. H. Kim and J. S. Lee, Small, 2017, 13, 1701751.

6 E. Y. Kim, D. Kumar, G. Khang and D. K. Lim, J. Mater. Chem. $B, 2015,3,8433-8444$.

7 H. Jung, J. Jung, Y. H. Kim, D. Kwon, B. G. Kim, H. B. Na and H. H. Lee, BioChip J., 2018, 12, 249-256.

8 J. Y. Noh, J. I. Kim, Y. W. Chang, J. M. Park, H. W. Song, M. J. Kang and J. C. Pyun, BioChip J., 2017, 11, 246-254.

9 M. Rana, M. Balcioglu, N. M. Robertson, M. S. Hizir, S. Yumak and M. V. Yigit, Chem. Sci., 2017, 8, 1200-1208.

10 L. A. Lane, X. Qian and S. Nie, Chem. Rev., 2015, 115, 1048910529.

11 P. Reineck, D. Gómez, S. H. Ng, M. Karg, T. Bell, P. Mulvaney and U. Bach, ACS Nano, 2013, 7, 6636-6648.

12 Y. Choi, T. Kang and L. P. Lee, Nano Lett., 2009, 9, 85-90.

13 M. Grzelczak, J. Pérez-Juste, P. Mulvaney and L. M. LizMarzán, Chem. Soc. Rev., 2008, 37, 1783-1791.

14 F. Kim, K. Sohn, J. Wu and J. Huang, J. Am. Chem. Soc., 2008, 130, 14442-14443.

15 N. D. Burrows, S. Harvey, F. A. Idesis and C. J. Murphy, Langmuir, 2017, 33, 1891-1907.

16 S. D. Perrault and W. C. W. Chan, J. Am. Chem. Soc., 2009, 131, 17042-17043.

17 Y. Zheng, Y. Ma, J. Zeng, X. Zhong, M. Jin, Z. Y. Li and Y. Xia, Chem.-Asian J., 2013, 8, 792-799.

18 Y. Zheng, X. Zhong, Z. Li and Y. Xia, Part. Part. Syst. Charact., 2013, 31, 266-273. 
19 Y. J. Lee, N. B. Schade, L. Sun, J. A. Fan, D. R. Bae, M. M. Mariscal, G. Lee, F. Capasso, S. Sacanna, V. N. Manoharan and G. R. Yi, ACS Nano, 2013, 7, 1106411070.

20 M. N. O'Brien, M. R. Jones, K. A. Brown and C. A. Mirkin, J. Am. Chem. Soc., 2014, 136, 7603-7606.

21 F. Shafiei, F. Monticone, K. Q. Le, X. X. Liu, T. Hartsfield, A. Alù and X. Li, Nat. Nanotechnol., 2013, 8, 95-99.

22 K. J. Park, J. H. Huh, D. W. Jung, J. S. Park, G. H. Choi, G. Lee, P. J. Yoo, H. G. Park, G. R. Yi and S. Lee, Sci. Rep., 2017, 7, 1557.

23 X. Ye, M. R. Jones, L. B. Frechette, Q. Chen, A. S. Powers, P. Ercius, G. Dunn, G. M. Rotskoff, S. C. Nguyen, V. P. Adiga, A. Zettl, E. Rabani, P. L. Geissler and A. P. Alivisatos, Science, 2016, 354, 874-877.

24 T. S. Deng, J. E. S. van der Hoeven, A. O. Yalcin, H. W. Zandbergen, M. A. van Huis and A. van Blaaderen, Chem. Mater., 2015, 27, 7196-7203.

25 A. Carattino, S. Khatua and M. Orrit, Phys. Chem. Chem. Phys., 2016, 18, 15619-15624.

26 C. M. Aguirre, T. R. Kaspar, C. Radloff and N. J. Halas, Nano Lett., 2003, 3, 1707-1711.

27 J. Rodríguez-Fernández, J. Pérez-Juste, P. Mulvaney and L. M. Liz-Marzán, J. Phys. Chem. B, 2005, 109, 14257-14261.
28 D. V. Talapin, A. L. Rogach, A. Kornowski, M. Haase and H. Weller, Nano Lett., 2001, 1, 207-211.

29 X. Xia, S. Xie, M. Liu, H. C. Peng, N. Lu, J. Wang, M. J. Kim and Y. Xia, Proc. Natl. Acad. Sci. U. S. A., 2013, 110, 66696673.

30 Y. Wang, J. He, C. Liu, W. H. Chong and H. Chen, Angew. Chem., Int. Ed., 2014, 54, 2022-2051.

31 S. K. Meena, S. Celiksoy, P. Schäfer, A. Henkel, C. Sönnichsen and M. Sulpizi, Phys. Chem. Chem. Phys., 2016, 18, 13246-13254.

32 J. Hassinen, V. Liljeström, M. A. Kostiainen and R. H. A. Ras, Angew. Chem., 2015, 127, 8101-8104.

33 Y. Lu, J. Y. Huang, C. Wang, S. Sun and J. Lou, Nat. Nanotechnol., 2010, 5, 218-224.

34 C. Zhu, H. C. Peng, J. Zeng, J. Liu, Z. Gu and Y. Xia, J. Am. Chem. Soc., 2012, 134, 20234-20237.

35 G. Dai, B. Wang, S. Xu, Y. Lu and Y. Shen, ACS Appl. Mater. Interfaces, 2016, 8, 13506-13511.

36 S. Yoon, B. Lee, C. Kim and J. H. Lee, Cryst. Growth Des., 2018, 18, 4731-4736.

37 J. Rodríguez-Fernández, J. Pérez-Juste, L. M. Liz-Marzán and P. R. Lang, J. Phys. Chem. C, 2007, 111, 5020-5025.

38 S. Yoon, B. Lee, J. Yun, J. G. Han, J. S. Lee and J. H. Lee, Nanoscale, 2017, 9, 7114-7123. 